\title{
Estimation earthquake occurrence probability in Kam- chatka region based on seismological and complex of ionospheric precursors
}

\author{
Vadim Boganov ${ }^{1, *}$ and Aleksey Pavlov ${ }^{1, * *}$ \\ ${ }^{1}$ Institute of Cosmophysical Research and Radio Wave Propagation FEB RAS, Paratunka, Russia
}

\begin{abstract}
In this paper the authors present the method of estimation of a region, waiting period and probability of strong earthquakes with $K_{S} \geq 13.5(M \geq 6.0)$ in Kamchatka region based on the combination of mid-term and short-term predictive signs accompanying earthquake formation. The seismological predictive parameter $\xi_{P}$ was taken as a mid-term precursor. It was calculated on the basis of the probabilistic model of seismic regime. A complex of ionospheric parameters was considered as short-term predictive signs with an earthquake waiting period of up to 5 days. It includes the K-layer, the sporadic Es layer of the $r$ type, the critical frequency $f \mathrm{oF} 2$, and the frequency stratification of the $\mathrm{F} 2$ layer. The probabilities of strong earthquakes with $K_{S} \geq 13.5(M \geq 6.0)$ that occurred over the period 2019-2021 in an expected zone, determined by the parameter $\xi_{P}$, were estimated on the basis of Bayes method provided that a complex of anomalous parameters of the ionosphere was identified.
\end{abstract}

\section{Introduction}

In [1], the results of ionospheric observations were presented for the purpose of short-term (3-5 days) assessment of the waiting period for Kamchatka earthquakes. At the same time, the application of the theoretical-probabilistic approach to the catalogue of Kamchatka earthquakes [2] allowed to calculate the probability of seismic events entering the seismofocal zone of the Kuril Islands and southern Kamchatka and to establish for it the growth of seismic activity that occurred during 2007-2013. This made it possible to make a medium-term forecast of major earthquakes that occurred in 2013 in this area. The task arises of developing a method for estimation the probability, area and time period of expectation of strong Kamchatka earthquakes with an energy class of $K_{S} \geq 13.5(M \geq 6.0)$ based on the joint of seismological and ionospheric prognostic signs based on the combination of seismological and ionospheric prognostic signs. This work is devoted to the solution of this problem.

\section{Method of calculation of seismological precursor of earthquakes}

Application of A. N. Kolmogorov's axiomatics to the catalogue of seismic events allows to consider each earthquake as an elementary event $\omega_{i}$, and the whole catalogue or its separate

\footnotetext{
*e-mail: vbogd@ikir.ru

**e-mail: pavlov@ikir.ru
} 
part as a space of elementary events $\Omega$ [2]. Each elementary event $\omega_{i}$ can be characterized by four random variables $\xi_{i}\left(\omega_{i}\right)=\varphi_{i}\left(\omega_{i}\right), \lambda_{i}\left(\omega_{i}\right), h_{i}\left(\omega_{i}\right), K_{i}\left(\omega_{i}\right)$, where quantities $\varphi_{i}, \lambda_{i}, h_{i}$ are the coordinates of the earthquake, and $K_{i}$ - energy class. (Event time $t_{i}\left(\omega_{i}\right)$, as having no mathematical expectation, is excluded from the system of random variables, but it is taken into account as a parameter that determines the occurrence of the event $\omega_{i}$ in the period under study $\Delta T)$. For a set of seismic events that occurred over a period of time $\Delta T$, the probability space is a collection of three objects $\{\Omega, \tilde{F}, P\}$, where $\Omega$ - space of elementary events, $\tilde{F}$ - set of subsets of random events, $P$ - probabilities of these events. Random events (for a specific selected period of time $\Delta T$ ) can represent an arbitrary combination of a variable number of random variables (when fixing others) and form some in $\tilde{F}$ subsets $A, B, C$ and etc.

The probability that a seismic event will fall within the specified intervals: latitude $\Delta \varphi=$ $\varphi_{i}-\varphi_{i-1}$, longitude $\Delta \lambda=\lambda_{j}-\lambda_{j-1}$, depth $\Delta h=h_{m}-h_{m-1}$ and energy class $\Delta K=K_{n}-K_{n-1}$

$$
\begin{array}{r}
P\left(\Delta \varphi_{i}, \Delta \lambda_{j}, \Delta h_{m}, \Delta K_{n}\right)=\int_{\varphi_{i-1}}^{\varphi_{i}} d \varphi \int_{\lambda_{j-1}}^{\lambda_{j}} d \lambda \int_{h_{m-1}}^{h_{m}} d h \int_{K_{n-1}}^{K_{n}} f(\varphi, \lambda, h, K) d K= \\
=F\left(\varphi_{i}, \lambda_{j}, h_{m}, K_{n}\right)-F\left(\varphi_{i-1}, \lambda_{j-1}, h_{m-1}, K_{n-1}\right)= \\
=P\left(\Delta \varphi_{i}\right) P\left(\Delta \lambda_{j} \mid \Delta \varphi_{i}\right) P\left(h_{m} \mid \Delta \varphi_{i}, \Delta \lambda_{j}\right) P\left(K_{n} \mid \Delta \varphi_{i}, \Delta \lambda_{j}, h_{m}\right)
\end{array}
$$

where $i, j, m$ and $n$-indices corresponding to intervals of random variables. Statistical processing of the catalog by the formula (1) makes it possible to calculate not only the average probability of occurrence of a seismic event in a given interval of geographical coordinates, depth and energy class through the corresponding conditional probabilities, but also to obtain numerical values of the step distribution function $F(\Delta \varphi, \Delta \lambda, \Delta h, \Delta K)$. As the number of events $n$ increases and the interval $\Delta$ decreases, the relative frequency $v$ tends to its mathematical counterpart $P$, and $F(\Delta \varphi, \Delta \lambda, \Delta h, \Delta K)$ - towards sustainable continuous distribution $F(\varphi, \lambda, h, K)$.

The random event $A$ on the basis of which the seismological precursor was calculated: «Hit of epicenters of seismic events that occurred in the selected volume $V$, in the specified intervals of latitude $\Delta \varphi$ and longitude $\Delta \lambda »$. The catalogue of earthquakes of Kamchatka Branch of Federal Research Center "United Geophysical Service RAS" during 1962-2021 was used for the analysis.

The studied seismically active area $S$ located along the Eastern coast of Kamchatka, including the Continental regions of Kamchatka, the Southern and Northern seismofocal zones is divided into $m=12$ non-overlapping areas with dimensions of $S_{i}=150 \times 200 \mathrm{~km}$ (Fig. 1). Since seismic events with energy class $K_{S}^{\mathrm{pr}} \geq 13.5$ are considered as predicted earthquakes in this paper, consequently, according to [3] the length of the gap in the focus of such earthquakes is $L \geq 11 \mathrm{~km}$. Assuming that the linear size of the earthquake preparation area is of the order of 10 rupture lengths, i.e. $110 \mathrm{~km}$, the spatial cell size of $150 \times 200 \mathrm{~km}$ can be chosen as the base case for calculations.

For each square $S_{i}(i=\overline{1, m})$ in a moving time window $\Delta T_{k}$ passing with step $\Delta t$, the time interval $T=1962-2021$ calculates the probability of seismic events $P_{k}\left(S_{i}\right)=n_{i k} / N_{k}$, where $n_{i k}$ - number of seismic events with energy class $K_{S} \geq 9.0$ and the depth of the hypocenter is less $200 \mathrm{~km}$, occurred in the area of $S_{i}$ for the period $\Delta T_{k}, N_{k}=\sum_{k=1}^{m} n_{i k}$ - total number of earthquakes with $K_{S} \geq 9.0$ and with the same hypocenter depth, took place on the square $S=\sum_{i=1}^{m} S_{i}$ during $\Delta T_{k}$. Also in each region $S_{i}$ long-term (background) values of probability of seismic events hit are calculated $P_{T}\left(S_{i}\right)=n_{i T} / N_{T}$, where $n_{i T}$ - number of seismic events with energy class $K_{S} \geq 9.0$, occured during $T, N_{T}=\sum_{k=1}^{m} n_{T k}$ - total number of earthquakes with $K_{S} \geq 9.0$, occurred during $T$ on the square $S$. In this paper, the size of the time window and its offset step are set equal, respectively $\Delta T_{k}=1$ year and $\Delta t=1$ day, a time interval at 


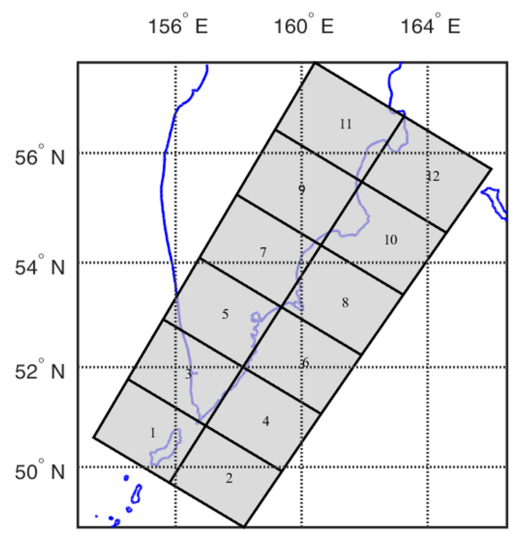

Figure 1. Division of the studied seismic area $S$ into unit cells $S_{i}$ with dimensions of $150 \times 200 \mathrm{~km}$.

which the study of the seismic regime is equal to $T=1962-2021$. On Fig. 2, as an example, the values of probabilities of seismic events in the region $S_{2}$ are presented.

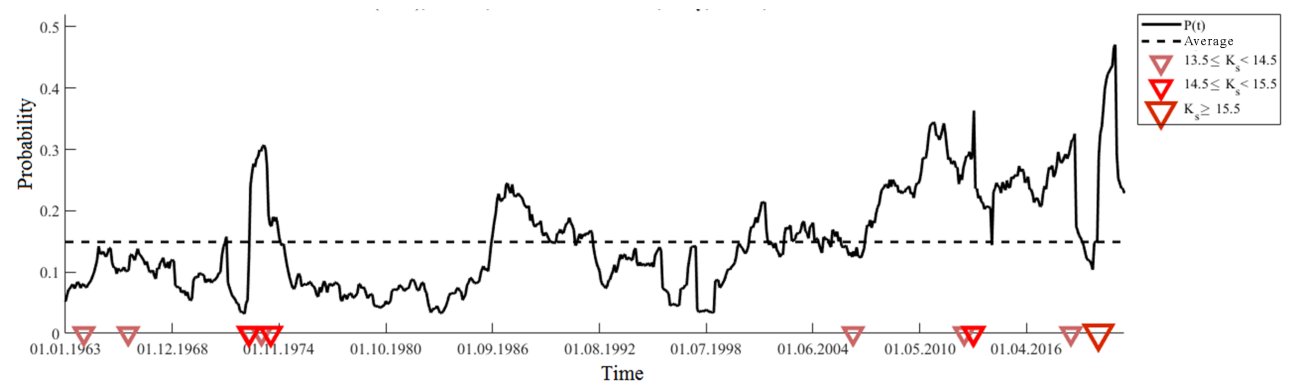

Figure 2. Time series of probability values $P_{k}\left(S_{2}\right)$. Red triangles on the time axis indicate earthquakes with $K_{S} \geq 13.5(M \geq 6.0)$.

To obtain statistically significant deviations of the current probability values $P_{k}\left(S_{i}\right)$ from their long-term (background) values $P_{T}\left(S_{i}\right)$ the parameter $\xi_{P}$, is used, determined on the basis of [4] and presented as:

$$
\xi_{P}= \begin{cases}\frac{\Delta P_{k}-\operatorname{sign}\left(\Delta P_{k}\right) \cdot\left|\sigma_{P_{k}}\right|}{\left|\sigma_{P_{T}}\right|}, & \text { if }\left|\Delta P_{k}\right|>\left|\sigma_{P_{k}}\right| \\ 0, & \text { if } 0 \leq\left|\Delta P_{k}\right| \leq\left|\sigma_{P_{k}}\right|\end{cases}
$$

where $P_{k}$ and $\sigma_{P_{k}}=\sqrt{\left(P_{k}\left(1-P_{k}\right) / n_{k}\right.}$ current probability values and its standard deviation in the sliding time window $\Delta T_{k} ; P_{T}$ and $\sigma_{P_{T}}=\sqrt{P_{T}\left(1-P_{T}\right) / n_{T}}$ long-term probability values and standard deviation $T>\Delta T_{k} ; n_{i}$ and $n_{T}$ - the number of seismic events that occurred over time periods, respectively $\Delta T_{k}$ and $T ; \Delta P_{k}=P_{k}-P_{T}$ the difference between the current $P_{k}$ probability value and the long-term probability value $P_{T}$. Fig. 3 presents the timing values parameter $\xi_{P}$ for region $S_{2}$.

Strong earthquakes can be preceded by periods of increased seismic activity (an increase in the number of background events), and periods of weakening of seismic activity (a decrease in the number of background events). When you search for periods of enhanced seismicity 


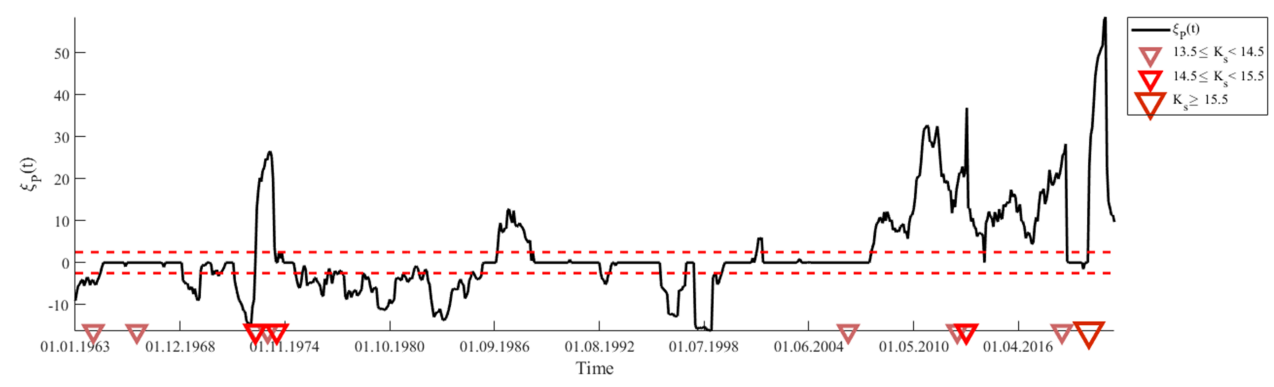

Figure 3. Temporary values of the parameter $\xi_{P}$ for region $S_{2}$. Red triangles on the time axis indicate earthquakes with $K_{S} \geq 13.5(M \geq 6.0)$.

Table 1. Predictive efficiency of the parameter $\xi_{P}$.

\begin{tabular}{|c|c|c|c|c|}
\hline Type of seismic anomaly & $V$ & $R$ & $J_{G}$ & $J_{M}$ \\
\hline Seismic activity $\left(\xi_{P} \geq 2.5\right)$ & 0.57 & 0.49 & 1.47 & 0.15 \\
\hline Seismic calm $\left(\xi_{P} \leq-2.5\right)$ & 0.51 & 0.52 & 2.15 & 0.28 \\
\hline Seismic activity and calm & 0.45 & 0.76 & 1.47 & 0.24 \\
\hline
\end{tabular}

in the beginning of the anomaly in the parameter $\xi_{P}$ is taken as the time of appearance of the values $\xi_{P} \geq \xi_{P}^{a}$, where $\xi_{P}^{a}$ - anxiety level is selected experimentally. In the case of searching for periods of seismic calm, the beginning of the anomaly is identified by the moment of occurrence of the values $\xi_{P} \leq \xi_{P}^{c}$, where $\xi_{P}^{c}$ - anxiety level is selected experimentally. Accordingly, when $\xi_{P}^{c}<\xi_{P}<\xi_{P}^{a}$ it is assumed that the current probability values do not significantly deviate from the background values. In this paper, the values of the criteria for the periods of activation and lull were established respectively $\xi_{P}^{a}=2.5$ and $\xi_{P}^{c}=-2.5$.

To assess the prognostic effectiveness of a prognostic feature $\xi_{P}$ following parameters are used: ratio of the number of earthquakes for which the precursor was allocated to the number of all earthquakes (reliability $R$ ), ratio of the number of precursor anomalies to the total number of isolated anomalies (validity $V$ ) [5], the effectiveness of A. A. Gusev [6] and G. M. Molchan's [7] methods.

The results of the evaluation of the predictive efficiency of parameter $\xi_{P}$ for earthquakes with $K_{S} \geq 13.5$ are presented in Table 1 and errors diagram (Fig. 4).

As can be seen from Table 1, the share of anomalies of seismic regime activation identified by parameter $\xi_{P}$ and preceding earthquakes (parameter $V$ ) was $57 \%$, and the share of calm anomalies was $51 \%$. The relative number of anomalies of the seismic regime of both types preceding the onset of earthquakes with $K_{S} \geq 13.5$, was $45 \%$. The activation of the seismic regime was preceded by $49 \%$ of earthquakes, and calm $-52 \%$. The $J_{G}$ prediction efficiency for both types of anomalies was 1.47. Since $J_{G}>1$, this predictive feature is considered informative and can be used in earthquake prediction algorithms. The $J_{M}$ prediction efficiency for both types of anomalies was 0.24 and as can be seen from the error diagram (Fig. 4) the values $(\tau, v)$ lie below the lower limit of $99 \%$ of the confidence interval (green curve), which can be interpreted as a high degree of reliability of the revealed connection between the identified anomalies of the seismic regime using the parameter $\xi_{P}$ and predicted earthquakes from the considered range of the energy class. 


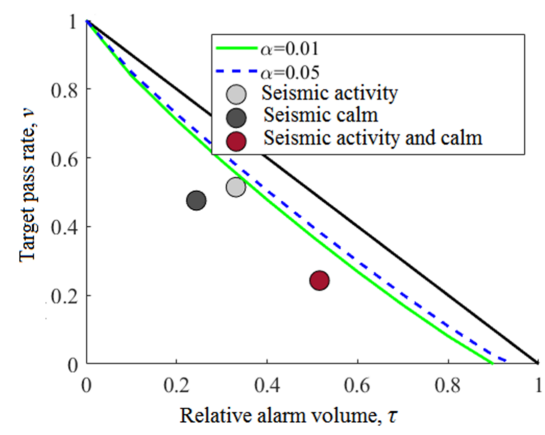

Figure 4. The diagram of errors for the parameter $\xi_{P}$ in predicting earthquakes with $K_{S} \geq 13.5$. The lower bounds of the confidence interval of the random forecast with the significance level are marked $\alpha=0.01$ and $\alpha=0.05$.

Table 2. The predictive efficiency of the complex ionospheric precursors.

\begin{tabular}{|c|c|c|c|c|c|c|c|c|c|}
\hline$n\left(E_{A}\right)$ & $n(E)$ & $n\left(A_{E}\right)$ & $n(A)$ & $V$ & $R$ & $J_{G}$ & $\tau$ & $v$ & $J_{M}$ \\
\hline 24 & 30 & 21 & 180 & 0.1 & 0.8 & 1.69 & 0.47 & 0.2 & 0.33 \\
\hline
\end{tabular}

\section{Method of short-term earthquake prediction based on the complex of ionospheric parameters}

In this work the data of radiophysical observations made by means of vertical radiosonding are used. The automatic ionospheric station (AIS) of vertical radiosonding is located in the village of Paratunka $\left(\varphi=52.97^{\circ} \mathrm{N}, \lambda=158.24^{\circ} \mathrm{E}\right)$. Observations are made once every 15 minutes in pulse mode at frequencies from 1 to $15 \mathrm{MHz}$.

The ionospheric precursors with an earthquake expectation period of up to 5 days were considered in article [8]. On the basis of the selected most effective ionospheric precursors, an algorithm for short-term earthquake prediction was constructed, in which a joint analysis of the ionospheric disturbances under consideration is carried out in a sliding time window of width $\Delta T=5$ days with a step $\Delta t=1$ day. The condition for announcing the start of the waiting period to seismic event was the execution in a time interval $\Delta T$ for at least three of the four ionospheric parameters consider the following criteria:

- K-layer formation during at least one day of $\Delta T$ interval;

- Formation of sporadic layer Es type $\mathrm{r}$ for at least one day interval $\Delta T$.

- Exceeding the critical frequency $f \mathrm{oF} 2$ layer F2 median values $f_{\text {med }}$, calculated for the previous 30 days, not less than $20 \%\left(\frac{f_{\mathrm{oF} 2}-f_{\text {med }}}{f_{\text {med }}} \geq 0.2\right)$ against the background of the development of the magnetospheric storm (total values of the $\mathrm{K}$-index per day $\Sigma K \geq 20$ );

- Stratification of the F2 layer in frequency (mode "V") for at least one day interval $\Delta T$;

The waiting period is chosen equal to $T_{\mathrm{w}}=5$ days. The algorithm was evaluated its predictive effectiveness in a time interval 01.01.2013-01.06.2021 for seismic events $K_{S} \geq$ $13.5(M \geq 6.0)$ at depths of $100 \mathrm{~km}$ in radius $r=500 \mathrm{~km}$ from the registration point of the ionospheric observations.

According to the results presented in Table 2, when predicting earthquakes with energy class $K_{S} \geq 13.5$, the reliability is 0.8 (i.e. $80 \%$ of earthquakes had a precursor), and the reliability is 0.1 (i.e. $10 \%$ of detected anomalies were realized). The efficiency value $J_{G}=1.69$ 


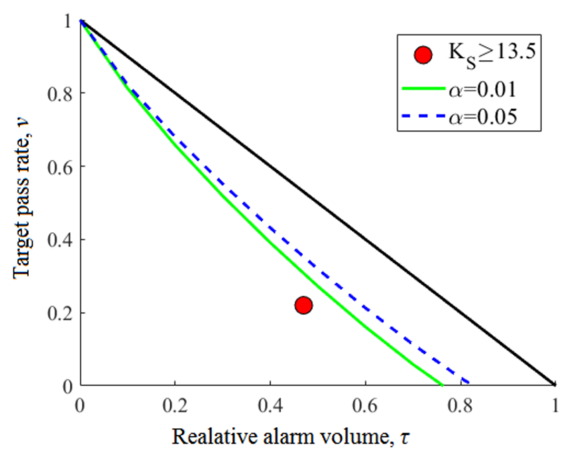

Figure 5. The diagram of errors for the algorithm of the complex ionospheric precursors for $K_{S} \geq$ 13.5.The lower bounds of the confidence interval of the random forecast with the significance level are marked $\alpha=0.01$ and $\alpha=0.05$.

shows that the prognosis by this method is statistically significant and 1.69 times different from random guessing. In the error diagram (Fig. 5) the values $(\tau, v)$ obtained for the range $K_{S} \geq 13.5$ lie below the lower limit of $99 \%$ of the confidence interval, which can be interpreted as a high degree of reliability of the revealed connection of the considered complex of ionospheric precursors with earthquakes of this range of energy class that occurred at distances up to $500 \mathrm{~km}$ from the observation point.

\section{Method to estimate earthquake occurrence probability}

The method to estimate the region, waiting time period and probability for earthquake with $K_{S} \geq K_{S}^{\mathrm{pr}}\left(K_{S}^{\mathrm{pr}}-\right.$ is the minimum energy class of the predicted earthquakes) based on a joint analysis of the seismic parameter $\xi_{P}$ and a complex of ionospheric precursors can be presented as follows:

1. Monitoring of the seismic regime in elementary cell $S_{i}$ based on the seismological parameter $\xi_{P}$. Area of expectations of strong earthquake will be considered $S_{\exp }$, which includes the unit cells, which was identified anomalies of seismic regime parameter $\xi_{P}$ : $S_{\exp }=\frac{1}{l} \sum_{j=1}^{l} S_{j}$, where $l \leq m$ - number of areas where abnormal parameter values are identified $\xi_{P}$. The average waiting period for a strong earthquake will be considered the period $\tilde{T}_{\mathrm{w}}=\frac{1}{l} \sum_{j=1}^{l} T_{j}^{\mathrm{w}}$, where $l$ - number of areas where abnormal parameter values are identified $\xi_{P}, T_{j}^{\mathrm{w}}=\tilde{T}_{j}^{\mathrm{w}}+\sigma_{T}$ - the waiting period earthquake in the cell $S_{j}$, $(j=\overline{1, l})$;

2. Monitoring of the ionospheric disturbances under consideration in a sliding time window of width $\Delta T=5$ days with a step $\Delta t=1$ day.

3. If the appearance of at least three of the four ionospheric parameters is recorded in the time interval $\Delta T$, then using formula 3 , the conditional probability $P\left(D_{1} \mid E I\right)$ of the onset of an earthquake in the area of expectations of strong earthquake $S_{\exp }$ is calculated.

4. If the probability $P\left(D_{1} \mid E I\right)$ exceed the specified criterion, then in the area $S_{\text {exp }}$, the waiting period $T_{\mathrm{w}}$ of a strong earthquake with a duration of 5 days is announced. The 
waiting period $T_{\mathrm{w}}$ can be extended if the complex of ionospheric precursors has been registered again.

The probability of occurrence of a seismic event with $K_{S} \geq K_{S}^{\mathrm{pr}}$ in the range $S_{\text {exp }}$ (random event $D_{1}$ ), provided that anomalies of the seismic regime are identified in it (random event $E$ ), as well as a complex of ionospheric precursors (random event $I$ ), calculated based on the Bayes formula:

$$
P\left(D_{1} \mid E I\right)=\frac{P\left(D_{1}\right) \cdot P\left(E \mid D_{1}\right) \cdot P\left(I \mid D_{1}\right)}{P\left(D_{1}\right) \cdot P\left(E \mid D_{1}\right) \cdot P\left(I \mid D_{1}\right)+P\left(D_{2}\right) \cdot P\left(E \mid D_{2}\right) \cdot P\left(I \mid D_{2}\right)}
$$

The random event $D_{1}$ - an earthquake occurrence predicted energy class $K_{S} \geq K_{S}^{\mathrm{pr}}$ in the range $S_{\text {exp. }}$.

The probability of this event is defined as $P\left(D_{1}\right)=\frac{n_{l}}{N}$, where $n_{l}=\sum_{j=1}^{l} n_{j}$ - the number of earthquakes with $K_{S} \geq K_{S}^{\mathrm{pr}}$ that occurred in the $S_{\exp }$ area during the observation period $T_{1}$, $N$ - the number of earthquakes with $K_{S} \geq K_{S}^{\mathrm{pr}}$ that occurred in the $S=\sum_{j=1}^{m} S_{j}$ area during the observation period $T_{1}$ of parameter $\xi_{P}$.

The random event $D_{2}-$ absence of earthquake with energy class $K_{S} \geq K_{S}^{\mathrm{pr}}$ in area $S_{\text {exp }}$. The probability of this event $D_{2}$ is $P\left(D_{2}\right)=1-P\left(D_{1}\right)$.

The random event $E$ - the occurrence of anomalous values of the parameter $\xi_{P}$.

The random event $I-$ the occurrence of complex of the ionospheric precursors. Then the events $E$ and $I$ can be represented in the form: $E I=D_{1} E I+D_{2} E I$

The random event $D_{1} E I$ - the occurrence of earthquake with $K_{S} \geq K_{S}^{\mathrm{pr}}$ in area $S_{\text {exp }}$ upon identification of an anomaly of the seismic regime (activation or calm) and the appearance of a complex of ionospheric precursors. The probability of this event is defined as $P\left(D_{1} E I\right)=$ $P\left(D_{1}\right) \cdot P\left(E I \mid D_{1}\right)=P\left(D_{1}\right) \cdot P\left(E \mid D_{1}\right) \cdot P\left(I \mid D_{1}\right)$.

The probability $P\left(E \mid D_{1}\right)=\frac{n_{\mathrm{pr} 1}}{N_{S_{\exp }}}$ is earthquakes occurrence probability with $K_{S} \geq K_{S}^{\mathrm{pr}}$ in area $S_{\text {exp }}$ when identifying seismic anomalies; $n_{\mathrm{pr} 1}$ - number of earthquakes with $K_{S} \geq K_{S}^{\mathrm{pr}}$, predicted when anomalies of the seismic regime appear over period $T_{1} ; N_{S_{\exp }}$ - total number of earthquakes with $K_{S} \geq K_{S}^{\mathrm{pr}}$ in area $S_{\exp }$ over period $T_{1}$.

The probability $P\left(I \mid D_{1}\right)=\frac{n_{\mathrm{pr} 2}}{N_{S_{\exp }}}$ is earthquakes occurrence probability with $K_{S} \geq K_{S}^{\mathrm{pr}}$ in area $S_{\text {exp }}$ when identifying the complex of ionospheric disturbances; $n_{\mathrm{pr} 2}-$ number of earthquakes with $K_{S} \geq K_{S}^{\mathrm{pr}}$, predicted when an ionospheric anomalies appears during the observation period $T_{2}\left(T_{2}\right.$ is a registration period of ionospheric parameters); $N_{S_{\text {exp }}}$ - total number of earthquakes with $K_{S} \geq K_{S}^{\mathrm{pr}}$ in area $S_{\text {exp }}$ over period $T_{2}$.

The random event $D_{2} E I-$ absence of earthquake with energy class $K_{S} \geq K_{S}^{\mathrm{pr}}$ in area $S_{\text {exp }}$ when identifying anomalies of the seismic regime (activation or calm) and appears complex of ionospheric precursors. The probability of this event is defined as $P\left(D_{2} E I\right)=P\left(D_{2}\right)$. $P\left(E I \mid D_{2}\right)=P\left(D_{2}\right) \cdot P\left(E \mid D_{2}\right) \cdot P\left(I \mid D_{2}\right)$.

The probability $P\left(E \mid D_{2}\right)=\frac{n_{\mathrm{fal}}}{N_{\mathrm{a} 1}}$ is probability of occurrence of abnormal parameter values $\xi_{P}$ (false alarms) in the area $S_{\text {exp }}$, which did not end with $K_{S} \geq K_{S}^{\mathrm{pr}}$ earthquakes; $n_{\mathrm{fa} 1}-$ number of false alarms per observation period $T_{1} ; N_{\mathrm{a} 1}$ - the total number of cases of abnormal behavior of the parameter $\xi_{P}$ in the area $S_{\text {exp }}$ over the period $T_{1}$.

The probability $P\left(I \mid D_{2}\right)=\frac{n_{\mathrm{fa} 2}}{N_{\mathrm{a} 2}}$ is probability of occurrence of ionospheric anomalies complex, which did not end with earthquakes; $n_{\mathrm{fa} 2}$ - number of false cases of the appearance of a complex of ionospheric parameters per observation period $T_{2} ; N_{\mathrm{a} 2}$ - the total number of occurrences of a complex of ionospheric parameters over the period $T_{2}$. 
Table 3. List of predicted earthquakes with energy class $K_{S} \geq 13.5(M \geq 6.0)$ over period 01.01.2019-01.06.2021.

\begin{tabular}{|c|c|c|c|c|c|}
\hline № & Date EQ & $K_{S}$ & $P\left(D_{1} \mid E I\right)$ & $S_{\exp }$ & $T_{\mathrm{w}}$ \\
\hline 1 & 2019.06 .25 & 14.3 & 0.69 & + & $2019.06 .21-2019.06 .26$ \\
\hline 2 & 2019.06 .26 & 14.4 & 0.69 & + & $2019.06 .21-2019.06 .26$ \\
\hline 3 & 2020.01 .22 & 14.3 & 0.71 & + & $2020.01 .21-2020.02 .05$ \\
\hline 4 & 2020.02 .20 & 14.3 & 0.72 & + & $2020.02 .11-2020.02 .29$ \\
\hline 5 & 2020.03 .25 & 16.8 & 0.73 & + & $2020.03 .19-2020.03 .28$ \\
\hline 6 & 2021.03 .16 & 14.6 & 0.74 & + & $2021.02 .14-2020.03 .30$ \\
\hline
\end{tabular}

\section{Retrospective predictive estimates of the area, period and probability of earthquake occurrence with $K_{S} \geq 13.5$}

In the considered seismically active region, 6 earthquakes with an energy class of $K_{S} \geq 13.5$ $(M \geq 6.0)$ occurred during the period 01.01.2019-01.06.2021. The results of the retrospective analysis according to the proposed method are presented in Table 3. Signs "+" and "-" observed earthquake respectively inside the waiting area $S_{\exp }$ and outside its borders. All six seismic events occurred during the waiting periods $T_{\mathrm{w}}$, determined from the complex of ionospheric parameters, and hit into the waiting area $S_{\text {exp }}$, determined from the values of the parameter $\xi_{P}$. The conditional probability $P\left(D_{1} \mid E I\right)$ of earthquake occurrence ranged from 0.69 to 0.74 .

Figure 6a shows, as an example, the waiting area for the earthquake of 25.03 .2020 with $K_{S}=16.8$, constructed on 19.03.2021 based on the analysis of the anomalous values of the parameter $\xi_{P}$.

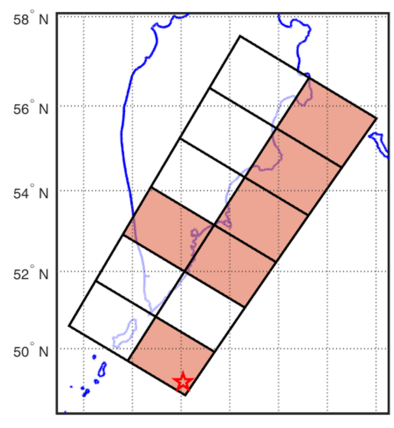

a)

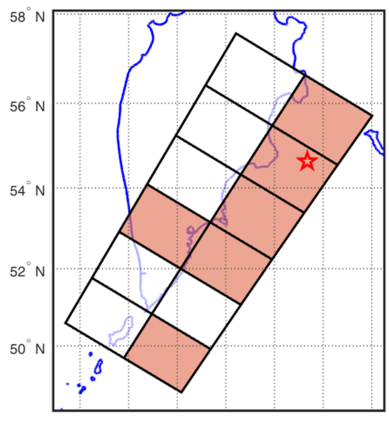

$154^{\circ} \mathrm{E} 156^{\circ} \mathrm{E} 158^{\circ} \mathrm{E} 160^{\circ} \mathrm{E} 162^{\circ} \mathrm{E} 164^{\circ} \mathrm{E} 166^{\circ} \mathrm{E}$

b)

Figure 6. Maps of expectation area $S_{\text {exp }}$ for earthquakes: a) 25.03.2020, $K_{S}=16.8$; b) 16.03.2021, $K_{S}=14.6$. Red stars on the map are earthquake epicenters.

The results of evaluating the effectiveness of earthquakes prediction with $K_{S} \geq 13.5$ for the time period 01.01.2019-01.06.2021, when choosing as the alarm level the values of the conditional probability $P\left(D_{1} \mid E I\right)=0.5$ and $P\left(D_{1} \mid E I\right)=0.7$, are presented in Table 4 and on the error diagrams (Fig. 7).

The analysis of the predictive efficiency of the presented method showed that at $P\left(D_{1} \mid E I\right) \geq 0.5$ its reliability $R=1$ (100\% of earthquakes were predicted), validity $V=0.09$ ( $9 \%$ of forecasts ended with earthquakes), efficiency $J_{G}$ was 1.8 (with $J_{G}=1$, the forecast 
Table 4. The effectiveness for the method of earthquakes prediction with $K_{S} \geq 13.5$ for chosen levels of the conditional probabilities $P\left(D_{1} \mid E I\right)$.

\begin{tabular}{|c|c|c|c|c|}
\hline Levels of conditional probability $P\left(D_{1} \mid E I\right)$ & $V$ & $R$ & $J_{G}$ & $J_{M}$ \\
\hline 0.5 & 0.09 & 1 & 1.8 & 0.44 \\
\hline 0.7 & 0.09 & 0.67 & 1.52 & 0.23 \\
\hline
\end{tabular}

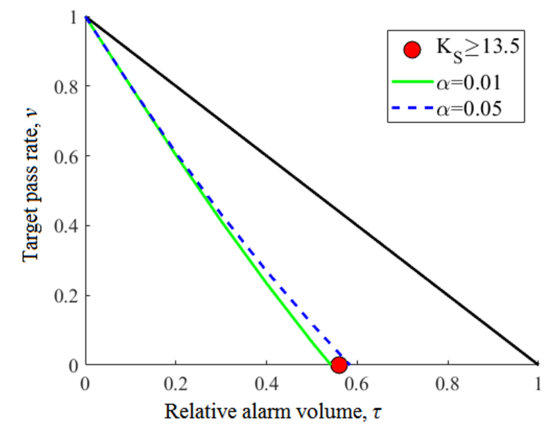

a)

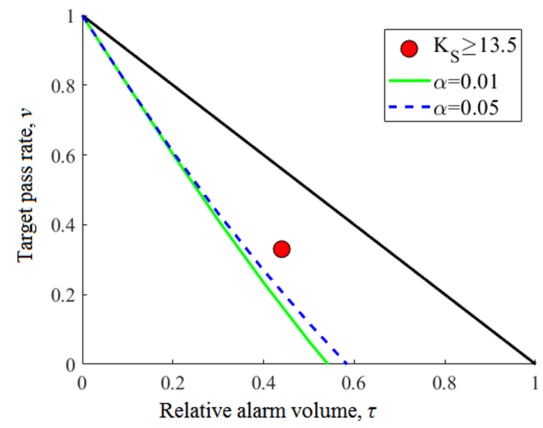

b)

Figure 7. The diagram of errors for the method of earthquakes prediction with $K_{S} \geq 13.5(M \geq 6.0)$ based on joint analysis of seismological and complex of ionospheric precursors for chosen levels of the conditional probabilities $P\left(D_{1} \mid E I\right)=0.5$ (a) and $P\left(D_{1} \mid E I\right)=0.7$ (b).

is considered random). The efficiency $J_{M}$ was 0.44 , which indicates the presence of a connection between the considered complex of precursors and earthquakes with an energy class $K_{S} \geq 13.5(M \geq 6.0)$.

\section{Conclusion}

An approach is presented that combines seismological and ionospheric prognostic signs in order to assess the probability of occurrence of earthquakes with $K_{S} \geq 13.5(M \geq 6.0)$ in the Kamchatka region. A retrospective analysis was carried out using this technique for the time period 01.01.2019-01.06.2021.

The effectiveness of method for estimation the area and possible period of the occurence of seismic events with $K_{S} \geq 13.5$ in the joint analysis of the seismic parameter $\xi_{P}$ and the complex of ionospheric precursors (at the values of the conditional probability of the onset of earthquakes $P\left(D_{1} \mid E I\right) \geq 0.5$ and $\left.P\left(D_{1} \mid E I\right) \geq 0.7\right)$ shows that the forecast using this method is more than 1.5 times different from a random guess.

The low validity of the $V$ forecast may be due to the fact that, ionospheric disturbances are formed against the background of solar activity, and it is not yet possible to accurately indicate the lower threshold of the earthquake magnitude, the preparation of which is already beginning to influence the state of the ionosphere. Seismic events with an energy class $K_{S} \geq 13.5$ predicted by ionospheric precursors and taken into account in statistics do not include events with an energy class $K_{S}<13.5$, although the ionospheric precursor has formed. Consequently, the ionospheric forecast signs that precede seismic events with $K_{S}<13.5$ are false for events with $K_{S} \geq 13.5$, thereby lowering the forecast reliability.

The work was carried out as part of the implementation of the state task AAAA-A21121011290003-0. 


\section{References}

[1] V.V. Bogdanov, A.V. Kaisin, A.V. Pavlov, A.L. Polyukhova, C.-V. Meister, J. Physics and Chemistry of the Earth 98, 154-160 (2017)

[2] V.V. Bogdanov, A.V. Pavlov, A.L. Polyukhova, J. Volcanol. Seismol. 4 (6), 412-422 (2010)

[3] Yu.V. Riznichenko, Issledovaniy po fizike zemletrjasenij (Nauka, Moskva, 1976)

[4] A.D. Zav'yalov, Prognoz zemletrjasenij (Nauka, Moskva, 2006)

[5] V.A. Saltykov, Physics of the Earth 2, 84-96 (2017)

[6] A.A. Gusev, Seismicity and seismic forecast, properties of the upper mantle and their relation to volcanism on Kamchatka (Nauka, Novosibirsk, 1974) 109-119

[7] G.M. Molchan, Physics of the Earth and Planetary Interiors 61, 84-98 (1990)

[8] V.V. Bogdanov, A.V. Pavlov, Vestnik KRAUNC. Fiz.-mat. nauki 30 (1), $59-78$ (2020). 